\title{
Zygomatic Arch Fracture Caused by a Flying Golf Ball: A Case Report
}

\author{
Kun Hwang ${ }^{1, *}$ \\ ${ }^{1}$ Department of Plastic Surgery, Inha University School of Medicine, Incheon, Korea \\ "Corresponding author: Department of Plastic Surgery, Inha University School of Medicine, 27, Inhang-ro, Jung-gu, 22332, Incheon, Korea. Email: jokerhg@inha.ac.kr
}

Received 2018 August 03; Revised 2019 February 12; Accepted 2019 March 14.

\begin{abstract}
Introduction: As golf becomes common, golf-related injuries become more widespread. Injury caused from hitting by a golf ball, resulting in a zygomatic arch fracture has not previously been described. In this report, zygomatic arch fracture caused by a golf ball is presented, and the basic mechanics of golf ball injuries are described.

Case Presentation: When a 38-year-old man was playing golf on a golf course, another player made a shot about $100 \mathrm{~m}$ behind him and the golf ball hit his left cheek. On physical examination, his left cheek was depressed and a contusion with a ball-mark was observed. His mouth opening was one and a half finger breadths. He felt pain and discomfort when clenching his jaw. Plain films and computed tomography of the facial bone showed a left zygomatic arch fracture. Under general anesthesia, the displaced fracture segments were reduced using a towel clip. Postoperatively, his mouth opening improved to 3 finger breadths.

Conclusions: In the field, serious face or head injuries on the golf course may be prevented by informing other golfers and shouting the word "fore (look ahead; a term warning the flight of a golf ball)" when it appears possible that a golf ball may hit other players or spectators. Other players or spectators should turn their back to the source of the call, duck, and cover their face and head with their hands. When meet the patients hit by a golf ball, surgeons should aware of the possibility of the facial bone fractures including nasal bone or zygoma.
\end{abstract}

Keywords: Golf, Zygoma, Fractures, Bone, Mechanics

\section{Introduction}

As golf has become more common, golf-related injuries, such as back pain and knee problems, have also become more widespread. However, facial bone fractures are very uncommon, especially zygomatic fractures.

The case of a zygomatic arch fracture caused by a golf ball is presented. This case is important as no such injury -in which an adult golfer was hit by a golf ball, resulting in a zygomatic arch fracture- has previously been described.

\section{Case Presentation}

A 38-year-old man was referred to the plastic surgery department from a local clinic. He had been hit by a golf ball on the left cheek. When he was playing golf on a golf course, his friend made a shot about $100 \mathrm{~m}$ behind him and a golf ball hit his left cheek (Figure 1). He did not lose consciousness.

On physical examination, his left cheek was found to be depressed and a contusion with a ball-mark was observed (Figure 2, upper left). His mouth opening was one and half finger breadths. He felt pain and discomfort when clenching his jaw. Otherwise, sensorimotor nerve damage was not detected.

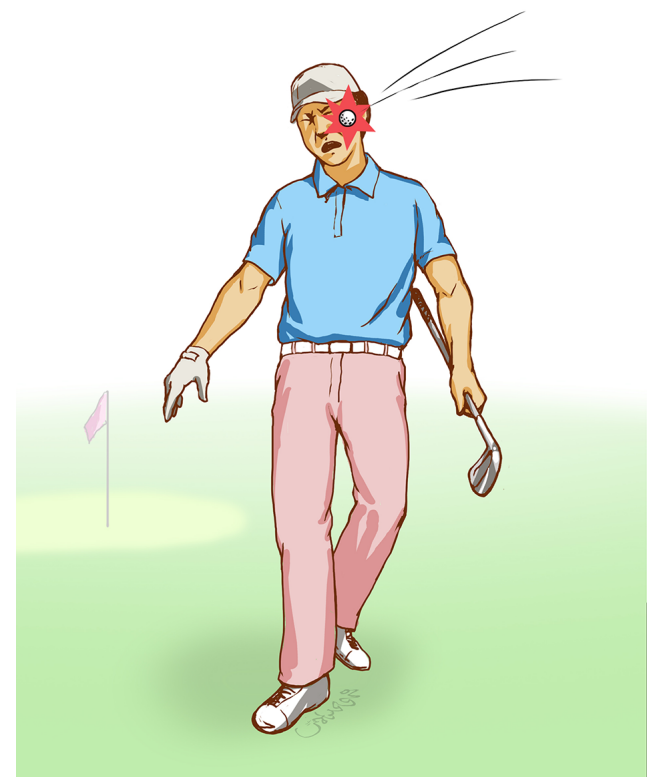

Figure 1. Mechanism of a golf ball hitting the cheek, causing a zygomatic arch fracture 

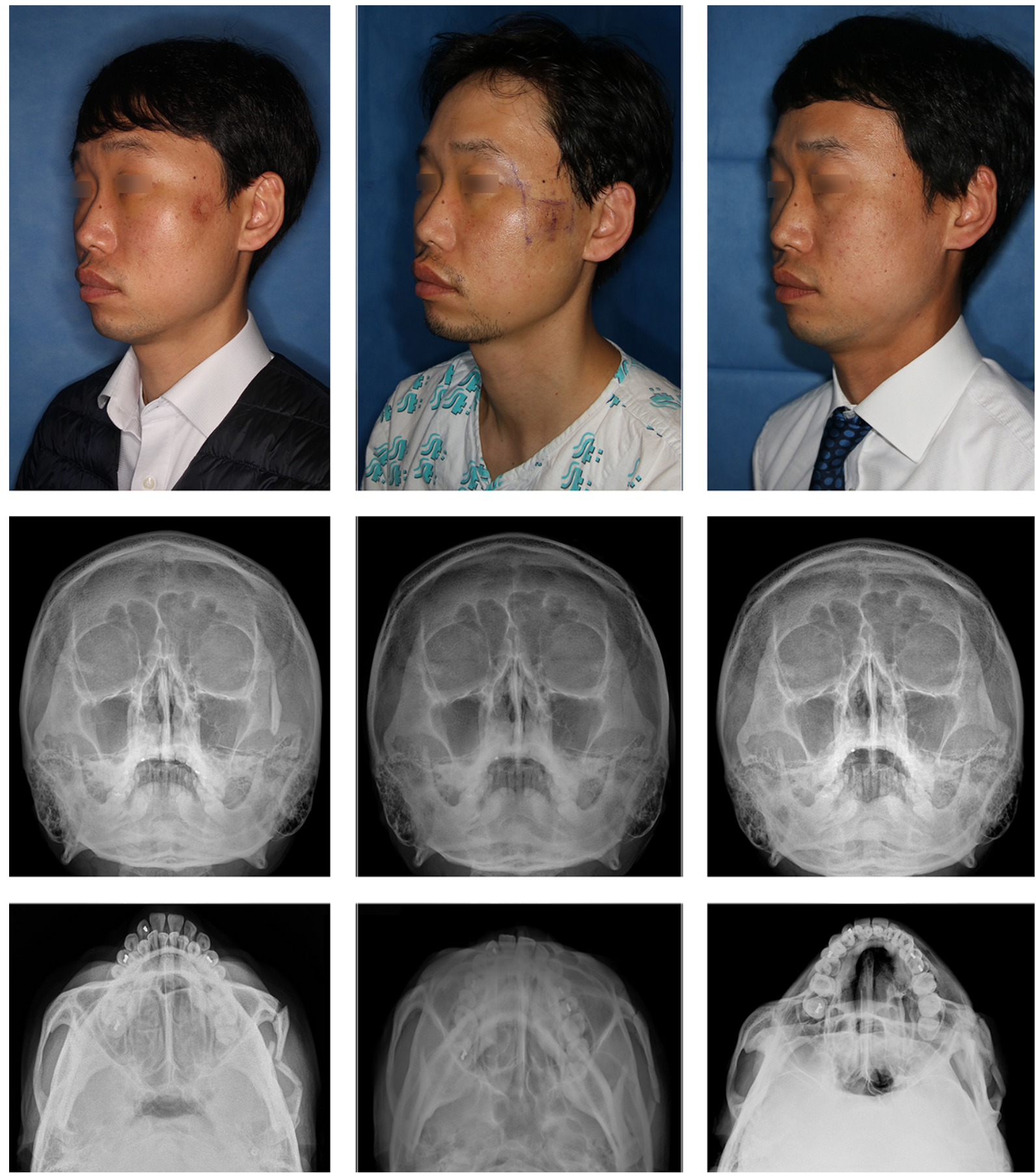

Figure 2. Photography and plain films of the 38-year-old man. Upper: photography, upper left: left cheek was depressed and a contusion with a ball-mark was observed, uppe center and right: the depressed left cheek improved after treatment; middle: Water's view; lower: zygomatico-axial view, left: pre-operation, center: post-operative 3 days, right: post-operative 6 months

Water's view and zygomatico-axial view showed a left zygomatic arch fracture (Figure 2, middle left, lower left).

Under general anesthesia, the displaced fracture seg- ments were reduced using a towel clip (1). With a marking pen, zygomatic arch and the orbital rim were outlined. By palpation, the fracture site was marked. The depressed 
fracture site was held with a towel clip and gently pulled outward direction. A click sound was heard. The clip was then released and removed. The contour of the zygomatic arch was compared with the other side.

The postoperative plain films revealed anatomical reduction of the segments (Figure 2, middle right, lower right).

Postoperatively, his mouth opening improved to 3 finger breadths (Figure 2, upper right).

\section{Discussion}

Facial injuries are not common in golfers. Most facial and head injuries are the result of being struck by a stray golf ball or by the head of a club used by a fellow golfer during the swing. This mechanism has the potential to cause a great amount of injury. Due to the size of the ball and its potential speed, it can cause considerable damage to the region that it hits (2).

According to the Golf Society of Great Britain, the golf ball is compressed against the club during the hit. Simultaneously the shaft on the club bends, which creates elastic kinetic energy. During the ball's flight, mechanical kinetic energy transfer continues, and makes the ball rise. Then the energy is transferred to the object that it eventually impacts $(3,4)$.

Under the rules of golf, a golf ball has a mass no more than $1.620 \mathrm{oz}(45.93 \mathrm{~g})$, and has a diameter not less than 1.680 in $(42.67 \mathrm{~mm})(5)$.

According to Newton's third law, the impulse (the force multiplied by the time for which the force is applied; joules) will cause the golf ball to speed up or slow down. For example, if the club applies a force of $9000 \mathrm{~N}$ for 0.0005 seconds on a ball with a mass of 0.045 kilograms, then the ball will be moving at 100 meters per second after the collision ( $9000 \mathrm{~N} \times 0.0005 \mathrm{~s} / 0.045 \mathrm{~kg})$.

In this case, a stray golf ball had enough energy to break the zygoma, a relatively thick and strong facial bone. As known, zygomatic arch fractures are classified as 3 types (Hönig Merten (HM) classification). This case belongs to type I (isolated tripod fracture) (6).

In this case, sensorimotor nerve damage was not detected. With such a high-velocity hit, why the facial nerve was not damaged? According to a recent paper of analyzing 495 maxillofacial trauma, incidence of nerve injury was $67.7 \%$. Among them, 8 patients (1.6\%) damaged facial nerve. The most commonly involved facial nerve branch was marginal mandibular, (5 patients, 1\%), and other branches (buccal, temporal, and zygomatic) were relatively rare (1 each, $0.2 \%$ each) (7). This less frequency of motor nerve injury might explain the intactness of zygomatic branch in this case.

Since the trigeminal nerve injury is relatively high in maxillofacial trauma (inferior alveolar branch; 39.1\%, in- fraorbital branch in 135 (27.2\%) (7), surgeons should check the sensory changes in the patients hit by a golf ball.

Serious face or head injuries on the golf course may be prevented by informing other golfers and shouting the word "fore (look ahead; a term warning the flight of a golf ball)" when it appears possible that a golf ball may hit other players or spectators. Other players or spectators should turn their back to the source of the call, duck, and cover their face and head with their hands. This prevents exposure of the face, eyes, and head to the flight of the ball (2).

When meet the patients hit by a golf ball, surgeons should aware of the possibility of the facial bone fractures including nasal bone or zygoma.

\section{Footnotes}

Conflict of Interests: The author has no conflict of interests to declare.

Funding/Support: This study was supported by a grant from National Research Foundation of Korea (NRF2019001834).

Patient Consent: Informed consent was obtained from the patient included in this study.

\section{References}

1. Hwang K, Lee SI. Reduction of zygomatic arch fracture using a towel clip. J Craniofac Surg. 1999;10(5):439-41. [PubMed: 10726515].

2. McHardy A, Pollard H, Luo K. Golf injuries: A review of the literature. Sports Med. 2006;36(2):171-87. doi: 10.2165/00007256-20063602000006. [PubMed: 16464124].

3. Cochran A, Stobbs J. The search for the perfect swing. New York: Lippincott; 1968.

4. Penner AR. The physics of golf. Rep Prog Phys. 2003;66(2):131-71. doi: 10.1088/0034-4885/66/2/202.

5. $R$ and A/USGA. Rules of golf and the rules of amateur status. London: Hamlyn; 2016.

6. Honig JF, Merten HA. Classification system and treatment of zygomatic arch fractures in the clinical setting. J Craniofac Surg. 2004;15(6):986-9. [PubMed: 15547388].

7. Poorian B, Bemanali M, Chavoshinejad M. Evaluation of sensorimotor nerve damage in patients with maxillofacial trauma: A single center experience. Bull Emerg Trauma. 2016;4(2):88-92. [PubMed: 27331065]. [PubMed Central: PMC4897989]. 